\title{
Assessment of the prevention and control effects on the outbreak of COVID-19 in Hunan, China: Based on a SEIAR Dynamic Model
}

\section{Wen-ting ZHA}

Hunan Normal University

\section{Nan ZHOU}

Hunan Normal University

Guoqun LI

Hunan Normal University

\section{Weitong LI}

Hunan Normal University

\section{Siyu ZHANG}

Hunan center for disease control and prevention

\section{Heng ZHANG}

Changsha center for disease control and prevention

\section{Mengxiang CHEN}

Hunan Normal University

\section{Ruihua FENG}

Hunan Normal University

\section{Tong LI}

Hunan Normal University

\section{Yi Su}

Hunan Normal University

yuan Iv ( $D$ ly598598@126.com )

Hunan Normal University

\section{Research article}

Keywords: SARS-CoV-2, COVID-19, SEIAR model, Regeneration number (Rt), Prevention and control measures

Posted Date: May 6th, 2020

DOI: https://doi.org/10.21203/rs.3.rs-20731/v1 
License: (c) (i) This work is licensed under a Creative Commons Attribution 4.0 International License. Read Full License 


\section{Title:}

Assessment of the prevention and control effects on the outbreak of COVID-19 in Hunan, China: Based on a SEIAR Dynamic Model

\section{Author names and affiliations:}

Wen-ting ZHA ${ }^{1}$, Nan ZHOU ${ }^{1}$, Guoqun $\mathrm{LI}^{1}$, Weitong $\mathrm{LI}^{1}$, Siyu ZHANG ${ }^{2}$, Heng ZHANG ${ }^{3}$, Mengxiang $\mathrm{CHEN}^{1}$, Ruihua FENG ${ }^{1}$, Tong $\mathrm{LI}^{1}$, Yi Su${ }^{1}$, Yuan $\mathrm{LV}^{1 *}$

1 Key Laboratory of Molecular Epidemiology of Hunan Province, School of Medicine, Hunan Normal University, Changsha, Hunan, People's Republic of China, 410081.

2 Hunan center for Disease Control and Prevention, Changsha, Hunan, People's Republic of China, 410005.

3 Changsha center for Disease Control and Prevention, Changsha, Hunan, People's Republic of China, 410004.

Wen-ting ZHA and Nan Zhou contributed equally to this work

\section{*Corresponding authors:}

\section{Yuan LV}

Key Laboratory of Molecular Epidemiology of Hunan Province, School of Medicine, Hunan Normal University, No. 371, Tongzipo Road, Yuelu District, Changsha, Hunan, People's Republic of China

Tel.: +86-15874019359

E-mail: 1y598598@126.com 


\section{ABSTRACT}

Background: A new infectious disease, Coronavirus disease 2019 (COVID-19) has been first reported during December 2019 in Wuhan, China, cases have been exported to other cities and abroad rapidly. Hunan is the neighboring province of Wuhan, a series of preventive and control measures were taken to control the outbreak of COVID-19. It is critical to assess these measures on the epidemic progression for the benefit of global expectation.

Method: A Susceptible-exposed-infections/asymptomatic-removed (SEIAR) model was established to evaluate the effect of preventive measures. Berkeley Madonna 8.3.18 was employed for the model simulation and prediction, and the curve-fitting problem was solved by Runge-Kutta fourth-order method.

Results: In this study, we found that $\mathrm{R}_{\mathrm{t}}$ was 2.71 from January 21 to 27 and reduced to 0.21 after January 27,2020 . If measures have not been fully launched, patients in Hunan would reach the maximum (8.96 million) on March 25, 2020, and end in about 208 days; when measures have been fully launched, patients in Hunan would just reach the maximum (699) on February 9, 2020, and end in about 56 days, which was very closed to the actual situation.

Conclusion: The outbreak of COVID-19 in Hunan, China has been well controlled under current measures, full implementation of measures could reduce the peak value, short the time to peak and duration of the outbreak effectively, which could provide a reference for controlling of COVID-19 for other countries. 
Keywords: SARS-CoV-2; COVID-19; SEIAR model; Regeneration number (Rt); Prevention and control measures.

\section{BACKGROUND}

A new infectious disease named Coronavirus disease 2019 (COVID-19), caused by Severe acute respiratory syndrome coronavirus 2 (SARS-CoV-2), has been first reported in Wuhan, China during December 2019 [1]. On January 23, 2020, Wuhan had been sealed off from outside, and the highest level of public health response to emergencies has been implemented in China. On January 31, the World Health Organization (WHO) announced that this outbreak of COVID-19 has become a public health emergency of international concern (PHEIC) [2]. As of April 14, 2020 Beijing time, there were just 2004 patients left and the outbreak of COVID-19 in China was gradually under control. But SARS-CoV-2 has spread rapidly over the world, 1917225 confirmed cases and 123401 deaths of COVID-19 have been reported in the world, including America $(\mathrm{N}=613886)$, Spain $(\mathrm{N}=174060)$, Italy $(\mathrm{N}=162488)$ and so on [3], which has caused a global pandemic.

Hunan is the neighboring province of Wuhan, China (Figure 1). Coinciding with Spring Festival travel rush, the period of mass migration in China, many people from Wuhan traveled to Hunan before the lockdown [4,5]. The first case of COVID-19 in Hunan was diagnosed on January 21, 2020, and the total number of COVID-19 in Hunan was 1018, ranking the 5th in provinces of China [3]. After the outbreak, a series of preventive and control measures like rigorous temperature monitoring, closing public 
spaces and entertainment, extending the national holiday, limiting travel and public gatherings, detection and treatment of patients, screening of close contacts, personal protection, publicity and education and so on were taken to control the spread of COVID-19. On February 29, 2020, there were no new cases in the outbreak in Hunan, and on March 14, 2020, there were no existing cases in Hunan anymore. The epidemic situation in Hunan has been well controlled, but we have never quantitatively evaluated the effectiveness of these measures, which is thus critical to assess the effects of these measures on the epidemic progression for the benefit of global expectation.

In this study, a Susceptible-exposed-infections/asymptomatic-removed (SEIAR) dynamic model was developed to estimate the transmissibility of SARS-CoV-2 and evaluate the effectiveness of prevention and control measures, to provide a reference for controlling the spread of COVID-19 for other countries.

\section{METHODS}

\section{Data source}

The reported case of COVID-19 in Hunan was collected from the Health Commission and the Center for Disease Control and Prevention of Hunan [6]. Migration data was collected from Baidu map insight--migration big data [7]. The epidemic curve from January 21 to March 14, 2020 was collected for our study, the simulation time step was one day.

\section{Prevention and control measures in Hunan}


After the outbreak, a series of unprecedented nationwide measures in Hunan were taken to control the spread of COVID-19 [6].

a) Active monitoring: Temperature monitoring points have been set up in airports, railway stations, passenger stations, wharves, high-speed entrances and exits, shopping malls, supermarkets, hotels, hospitals, residential areas and so on, people with abnormal temperature would be isolated and observed immediately. For the migratory person, especially those who just came from Wuhan or other cities of Hubei province, doctors would observe them for 14 days and measure their temperature twice a day to find out whether they have fever, cough, chest distress or other symptoms that relevant to this disease, once relevant symptoms has been found, they would be sent to medical institutions for isolation and treatment.

b) Environmental Management: Temporarily close cultural, tourism and entertainment places such as scenic spots, cinemas, internet cafes, and other public spaces. Extend the national holiday, limit travel and encourage people to stay at home, reduce large-scale public gathering activities and meetings. Clean up key places such as residential quarters and garbage transfer stations, dispose garbages timely.

c) Detection and treatment of patients: 116 designated hospitals were identified in Hunan, case diagnosis procedures were formulated in time to improve the efficiency of diagnosis and triage in medical institutions. Scientists in China have tried their best to develop effective drugs and treatments as soon as possible, and doctors have tried their best to detect and treat patients, especially for severe patients;

d) Screening of close contacts: Close contacts with COVID-19 patients were screened 
through epidemiological investigation and big data information technology. Family doctors would manage the close contacts through guiding them to carry out home isolation. Medical observation, nucleic acid diagnosis, isolation, and treatment would be carried out once abnormal conditions were found.

e) Personal protection: People are recommended to wear masks when going out and wash hands when entering home. It is suggested to reduce large-scale gathering and visiting each other, maintain indoor sanitation and air circulation, do not or less go to crowded public places in case of cross infection. If there were fever or other respiratory tract infection symptoms, go to medical institutions in time.

f) Publicity and education: Knowledge of COVID-19 were spread through television, broadcast, WeChat, mobile short messages or other forms to arouse the awareness of public and enhance their preventive ability.

g) Hotline: A hotline has also been opened in Hunan to provide knowledge consultation about COVID-19 and psychology services under this special circumstances.

\section{Models and statistical analysis}

\section{SEIAR model}

A SEIAR model was established to evaluate the effect of prevention and control measures in Hunan. According to the disease status, people were divided into five departments, susceptible people(S), exposed people (E), symptomatic infected people (I), asymptomatic infected people (A) and removed people(R).[8,9]. The model was 
developed based on the following facts or assumptions: 1) $\mathrm{S}$ was assumed to have an equal infectious rate $(\beta)$ with $\mathrm{I}$, the ratio of infectious rate of A to I was $\kappa ; 2$ ) E would turn to I or A after a certain exposed period $(1 / \omega)$, the latency coefficient $(\omega)$ meant the rate each E turn to I or A per unit day; 3) $1 / \gamma$ was the infectious period of I or A, $\gamma$ meant the removal rate, the number of I and A turn to $R$ per unit time was $\left.\left(\gamma_{1} A+\gamma_{2} I\right) ; 4\right)$ The deaths of COVID-19 were ignored because it was very low in Hunan $[8,9]$.

$d \mathrm{~S} / d \mathrm{t}, d \mathrm{E} / d \mathrm{t}, d \mathrm{t} / d \mathrm{t}, d \mathrm{~A} / d \mathrm{t}$ and $d \mathrm{R} / d \mathrm{t}$ indicated the number of individuals (n) at time $\mathrm{t}$ in the corresponding departments, respectively, the model equations were as follows:

$$
\left\{\begin{array}{l}
d S / d t=-\beta S I-\kappa \beta S A \\
d E / d t=\beta S I+\kappa \beta S A-p w E-(1-p) w E \\
d A / d t=p w E-\gamma_{1} A \\
d I / d t=(1-p) w E-\gamma_{2} I \\
d R / d t=\gamma_{1} A+\gamma_{2} I
\end{array}\right.
$$

\section{Parameter estimation}

There were five initial values and six parameters in the model, which were listed in Table 1.

a) By the end of the year 2019, the population of Hunan was 69 million [10], $\mathrm{S}_{0}=69183800$

b) According to Baidu migration big data in China, there were about 250 thousand people traveled from Wuhan to Hunan one week before the lockdown[7], and the incidence rate of COVID-19 in Wuhan at that time was about $0.1 \%[11,12]$, it was estimated that exposed people entered Hunan at that time was $\mathrm{E}_{0}=250$

c) The mean incubation period $(1 / \omega)$ was 5.2 days (95\% CI: $4.1-7.0)$ [13], $\omega=1 / 5.2$. 
d) As of February 12, 2020, 972 symptomatic infectious people and 121 asymptomatic infectious people were reported in Hunan, so the proportion of asymptomatic infectious rate of people in the model was $P=121 /(972+121)=11.1 \%$.

e) Once symptomatic infected individuals were diagnosed, they would be isolated, and the average time from onset to diagnosis was 3 days in Hunan, so, the removal rate of I was $\gamma_{2}=1 / 3$; While those asymptomatic infected individuals would not be easily found and isolated, the recovery day of A was 14 days [14], so the removal rate of A was $\gamma_{1}=1 / 14$.

\section{Simulation methods}

Data from the outbreak of COVID-19 in Hunan was fitted to a SEIAR model curve to estimate $\beta$ and $\kappa$ in different periods. Berkeley Madonna 8.3.18 and Microsoft Office Excel 2010 software were employed for the model simulation and data management, respectively. Graphpad prism 5 was used for the figure development, while the curve-fitting problem was solved by the Runge-Kutta fourth-order method, with a tolerance of 0.001 . A Goodness of fit test ( $\chi^{2}$ test) was performed using the IBM-SPSS software, in which the significance level was $\alpha=0.05$.

\section{Evaluation about the effectiveness of prevention and control measures}

Based on the implementation degree of the prevention and control measures in Hunan, we divided the time into two periods. The first period was from January 21 to 27 , 2020, when these measures have not been fully launched; the second period was from January 28 to March 14, 2020, during which these measures have been fully launched.

a) The effects on Rt 
The regeneration number $\left(\mathrm{R}_{\mathrm{t}}\right)$ meant the expected number of secondary infections which result from introducing a single infected individual into an otherwise susceptible population at the time $t$ [15]. Calculate $R_{t}$ in these two periods to evaluate the prevention and control effect in Hunan. If $R_{t}>1$, the outbreak would continue, if $R_{t}<1$, the outbreak would go to end, the more reduction of $R_{t}$, the better the control effect. $R_{t}$ was calculated by the following formula [13]:

$$
R_{t}=\beta S_{t}\left(\frac{1-p}{\gamma_{2}}+\frac{\kappa p}{\gamma_{1}}\right)
$$

b) The effects on peaks and epidemic duration

Two prediction models based on different parameters obtained by model fitting in different periods were established, the peaks and epidemic duration in two models were compared to explore whether the full implementation of prevention and control measures has an impact on the peak value, peak time and duration of the epidemic.

\section{RESULTS}

\section{Epidemiological features of the outbreak of COVID-19 in Hunan}

The first case of COVID-19 in Hunan was diagnosed on January 21, 2020, the cumulative patients of COVID-19 reached the maximum value of 698 on February 10, and the outbreak of COVID-19 in Hunan ended at March 14, when there were no existing native cases anymore. From January 21 to March 14, 2020, 1018 patients of COVID-19 in Hunan were reported, and 4 have died. Changsha, the capital of Hunan, reported the largest number of patients (242 cases, accounting for 23.8\%), followed by Yueyang (156 cases, 15.3\%) and Shaoyang (102 cases, 10.0\%). The distribution of time 
and spaces among patients in Hunan was shown in Figure 3.

\section{The effects of measures on Rt}

The results of curve fitting of the data in Hunan and SEIAR model showed that the simulated result agreed well with the reported data when $\beta=1.10 \times 10^{-8}$ and $\kappa=0.83$ from January 21 to $27,2020, \quad \beta=1.14 \times 10^{-9}$ and $\kappa=0.84$ from January 27 to March 14 , 2020. $R_{t}$ equalled to 2.71 in the first period, and reduced to 0.21 in the second period when measures have been fully launched. The model fitting diagram was shown in Figure 4.

\section{The effects of measures on peaks and epidemic duration}

The prediction results showed that if prevention and control measures have not been fully launched, the total number of patients in Hunan would reach the maximum (8.96 million patients) on the 64th day after the outbreak (March $25^{\text {th }}, 2020$ ), the outbreak would end in about 208 days (August 16 ${ }^{\text {th }}, 2020$ ); but if prevention and control measures have been fully launched, the total number of patients in Hunan would reach the maximum (699 patients) on the 19th day after the outbreak (February $9^{\text {th }}, 2020$ ), the outbreak would end in about 56 days (March $17^{\text {th }}, 2020$ ), which was fitting well with the actual data $\left(\mathrm{R}^{2}=0.957, P<0.001\right)$. The forecast results were shown in Figure 5 .

\section{DISCUSSION}

It is important to decrease $\mathrm{R}_{\mathrm{t}}$ to control the transmission of virus [14-18]. Due to different time and places, $\mathrm{R}_{\mathrm{t}}$ obtained by different researchers were ranged from 1.10 to 
6.47 in China [14-18]. The number of patients is changing rapidly, the measures taken in different time and places are not all the same, which make it necessary to adjust the model and parameters in respect of different situations in different regions. In this study, we found that $R_{t}$ was 2.71 from January 21 to 27 in Hunan, which was similar to $R_{0}$ in the early stage of Wuhan $[14,16,18]$, and reduced to 0.21 rapidly after January 27,2020 , which means that the prevention and control measures in Hunan were very effective in controlling the spread of SARS-CoV-2 and the outbreak of COVID-19 in Hunan has been well controlled by current measures.

The prediction results showed that the cumulative patients of COVID-19 in Hunan would reach the maximum value of 699 on February 9, and the outbreak would end around March 17, which fits very well to the actual data. Compared with the early stage, with the full implementation of prevention and control measures, it has reduced the peak value, shortened the time to peak and duration of the outbreak effectively, which lessened the harm brought by the outbreak in Hunan, and provided a reference for controlling of COVID-19 for other countries.

In addition, we also found that the proportion of asymptomatic infected people of COVID-19 was low in Hunan $(\mathrm{P}=11.1 \%)$, but the infection rate were just little lower than symptomatic infected people ( $\kappa=0.83$ to 0.84 ). In the follow-up work, we should pay more attention to asymptomatic infected people and carry out effective methods to find out them, especially from the focus groups, such as employees and students returning to enterprises or schools from Wuhan or abroad, close contacts of confirmed patients, medical workers and so on to reduce the impact of asymptomatic infection on 
the re-outbreak of COVID-19.

The actual situation was changeable and complex, differences remained between the simulation and actual data, which is the limitation of our study. Besides,

the number of imported COVID-19 cases in China is increasing with the rapid growth of cases abroad, which may have some impact on the development of the epidemic of COVID-19 in China, the model and parameters need further adjustment according to the actual situation.

\section{CONCLUSIONS}

The outbreak of COVID-19 in Hunan, China has been well controlled by current measures, full implementation of measures could reduce the peak value, short the time to peak and duration of the outbreak effectively, which could provide a reference for controlling of COVID-19 for other countries.

\section{List of abbreviations}

1. Coronavirus disease 2019 (COVID-19)

2. Severe acute respiratory syndrome coronavirus 2 (SARS-CoV-2)

3. World health organization (WHO)

4. Public health emergencies of international concern (PHEIC)

5. Susceptible-exposed-infections/asymptomatic-removed (SEIAR)

6. Regeneration number $(\mathrm{Rt})$

7. Confidence interval $(C I)$ 


\section{Declarations}

\section{Ethics and Consent to Participate}

This article is a research with mathematical models using publically available data (http://wjw.hunan.gov.cn/wjw/qlzhyqfkgz/yqfkgz.html), ethics is not applicable.

All authors agreed to participate in this study, the consent obtained from study participants was written.

\section{Consent to publish}

All authors agreed to publish this article on BMC public health

\section{Availability of data and materials}

All data and material in our study were publically available.

The reported case of COVID-19 in Hunan were collected from the Health Commission and Center for Disease Control and Prevention of Hunan, http://wjw.hunan.gov.cn/wjw/qlzhyqfkgz/yqfkgz.html. Migration data was collected from Baidu map insight--migration big data, http://qianxi.baidu.com.

\section{Competing interests}

The authors declare that they have no competing interests.

\section{Funding}


The work was supported by Education Project of Hunan Provincial Department [2017] No. 451, [2017] No. 452 and JG2018B041.

The funder had in our study was the correspondence author (Yuan LV).

\section{Authors' Contribution}

W Z mainly responsible for Writing - original draft, review \& editing;

N Z mainly responsible for Methodology;

G L and W L mainly responsible for Software;

H Z, S Z, M C and R F mainly responsible for Resources of data and Data curation;

T L and Y S mainly responsible for Formal analysis;

Y L mainly responsible for sponsorship of Funding acquisition and Supervision.

All authors read and approved the final manuscript.

\section{Acknowledgements}

We are grateful to everyone who helped us to complete this study successfully.

\section{REFERENCES}

[1]World Health Organization. Coronavirus. World Health Organization, cited January 19, 2020. Available:309. https://www.who.int/health-topics/coronavir.

[2]World Health Organizaiton. Statement on the second meeting of the International HealthRegulations Emergency Committee regarding the outbreak of novel coronavirus (2019-nCoV). https://www.who.int/news-room/detail/30-01-2020 statement on the 
second meeting of the international health regulations.

[3]Chinese Center for Disease Control and Prevention. Distribution of COVID-19. http://2019ncov.chinacdc.cn/2019-nCoV.

[4] 2019 Data from spring festival (in Chinese) 2019. Available online:

http://news.sina.com.cn/c/2019-02-04/docihrfqzka3579637.shtml

[5]Isaac I. Bogoch, Alexander Watts, Andrea Thomas-Bachli, et al. Pneumonia of unknown aetiology in Wuhan, China: potential for international spread via commercial airtravel. Journal of Travel Medicine, 2020; 1-3. http://doi: 10.1093/jtm/taaa008.

[6]The Health Commission of Hunan. The notification of COVID-19.

http://wjw.hunan.gov.cn/wjw/qlzhyqfkgz/yqfkgz.html

[7]Baidu map insight. The migration big data. http://qianxi.baidu.com.

[8]Wen-ting ZHA, Feng-rui PANG, Nan ZHOU, et al. Research about the optimal strategies for prevention and control of varicella outbreak in a school in a central city of China: based on an SEIR dynamic model. Epidemiology and Infection, 2020; 148(56): 1-8. https://doi.org/10.1017/S0950268819002188

[9]Wen-ting ZHA, Nan ZHOU, Guoqun LI, et al. Assessment and forecasting the spread of SARS-CoV-2 outbreak in Changsha, China: Based on a SEIAR Dynamic Model. Research square (Preprint), 2020, 03:1-12.

[10]Hunan Provincial Bureau of Statistics. Hunan statistical yearbook-2019. http://tji.hunan.gov.cn/hntj/tjsj/tjnj/index.html

[11]Shi Zhao, Salihu S. Musa, Qianying Lin, et al. Estimating the Unreported Number of Novel Coronavirus (2019-nCoV) Cases in China in the First Half of January 2020: A 
Data-Driven Modelling Analysis of the Early Outbreak. Journal of clinical medicine, 2020; 9, 388. http://doi:10.3390/jcm9020388.

[12]T. Chen, R. Ka-Kit Leung, R. Liu, F. Chen, X. Zhang, J. Zhao, S. Chen, Risk of imported Ebola virus disease in China. Travel medicine and infectious disease 12, 650-658 (2014); published online EpubNov-Dec (10.1016/j.tmaid.2014.10.015)

[13]Qun Li, M. Xuhua Guan, Peng Wu,et al. Early Transmission Dynamics in Wuhan, China, of Novel Coronavirus-Infected Pneumonia. N Engl J Med, 2020; published online Jan 24. http://doi:10.1056/NEJMoa2001316.

[14]Joseph $\mathrm{T} \mathrm{Wu}$, Kathy Leung, Gabriel M Leung. Nowcasting and forecasting the potential domestic and international spread of the 2019-nCoV outbreak originating in Wuhan, China: a modelling study. Lancet, 2020, published online January 31. http://doi.org/10.1016/S0140-6736(20)30260-9.

[15]Li Liming, Liang Xiaofeng, Jiang Qingwu, et al. Latest understanding of the epidemiology of COVID-19. Chinese Journal of epidemiology, 2020; 41 (2): 139-144. [16]Isaac I. Bogoch, Alexander Watts, Andrea Thomas-Bachli, et al. Pneumonia of unknown aetiology in Wuhan, China: potential for international spread via commercial airtravel. Journal of Travel Medicine, 2020; 1-3. http://doi: 10.1093/jtm/taaa008. [17]Jonathan M. Read, Jessica R.E. Bridgen, Derek A.T. Cummings, Antonia Ho, Chris P. Jewell. Novel coronavirus NCP: early estimation of epidemiological parameters and epidemic predictions. MedRxiv preprint posted online, Jan 27, 2020.https://doi.org/10. $1101 / 2020.01 .23 .20018549$. 
[18]Tianmu Chen, Jia Rui, Qiupeng Wang, et al. A mathematical model for simulating the transmission of Wuhan novel Coronavirus. BioRxiv preprint posted online, Jan 19, 2020. http://doi: 10.1101/2020.01.19.91166.

\section{Tables and Figures}

Table 1 List of parameters and initial values of each categorie in model

Figure 1 The geographical location map of Hunan

Figure 2 Flow chart of SEIAR model of COVID-19

Figure 3 The distribution of time and space among patients in the outbreak of COVID-19 in Hunan

Figure 4 The result of curve fitting of actual data and SEIAR model

Figure 5 The forecast results with different parameters

(Note: 5A The forecast with parameters obtained by model fitting from January 21 to 27 , 2020, when meansures have not been fully launched; 5B The forecast with parameters obtained by model fitting from January 28 to March 14, 2020, when meansures have been fully launched ) 
Figures



\section{Figure 1}

The geographical location map of Hunan Note: The designations employed and the presentation of the material on this map do not imply the expression of any opinion whatsoever on the part of Research Square concerning the legal status of any country, territory, city or area or of its authorities, or concerning the delimitation of its frontiers or boundaries. This map has been provided by the authors.

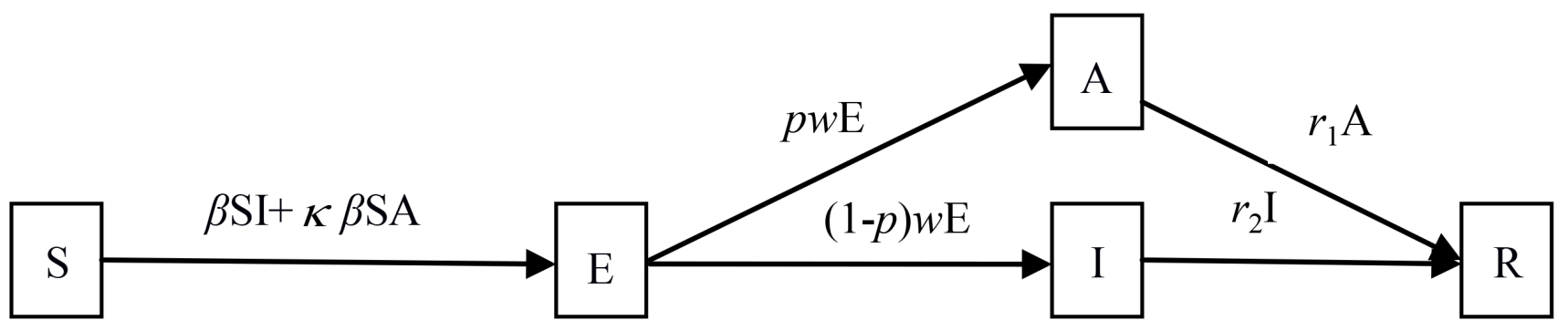

Figure 2

Flow chart of SEIAR model of COVID-19 

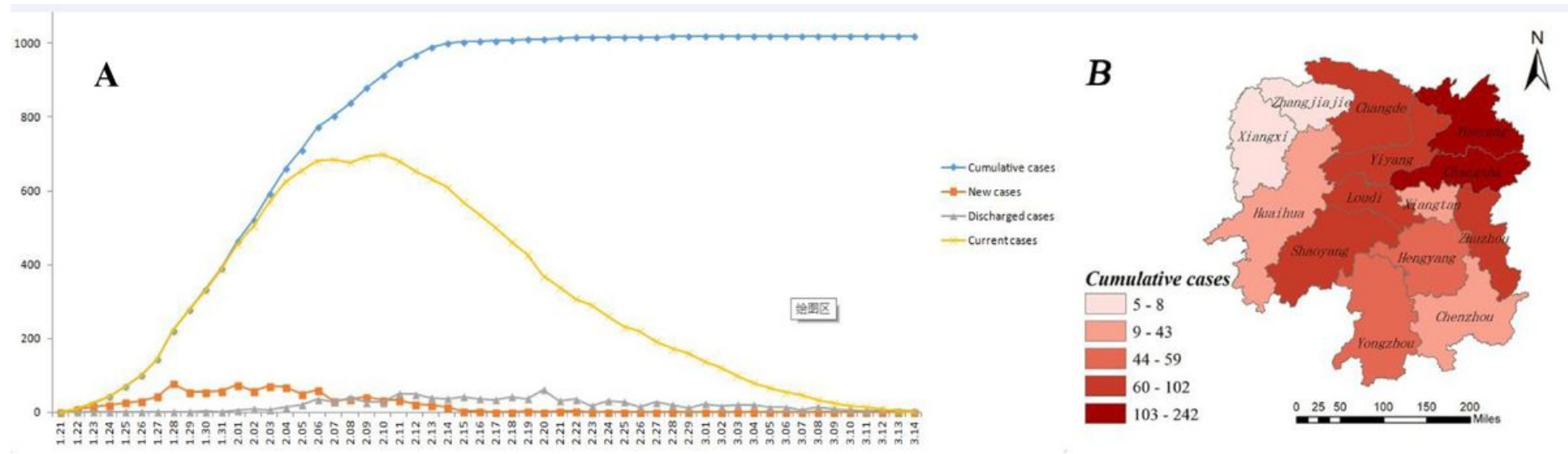

\section{Figure 3}

The distribution of time and space among patients in the outbreak of COVID-19 in Hunan Note: The designations employed and the presentation of the material on this map do not imply the expression of any opinion whatsoever on the part of Research Square concerning the legal status of any country, territory, city or area or of its authorities, or concerning the delimitation of its frontiers or boundaries. This map has been provided by the authors.

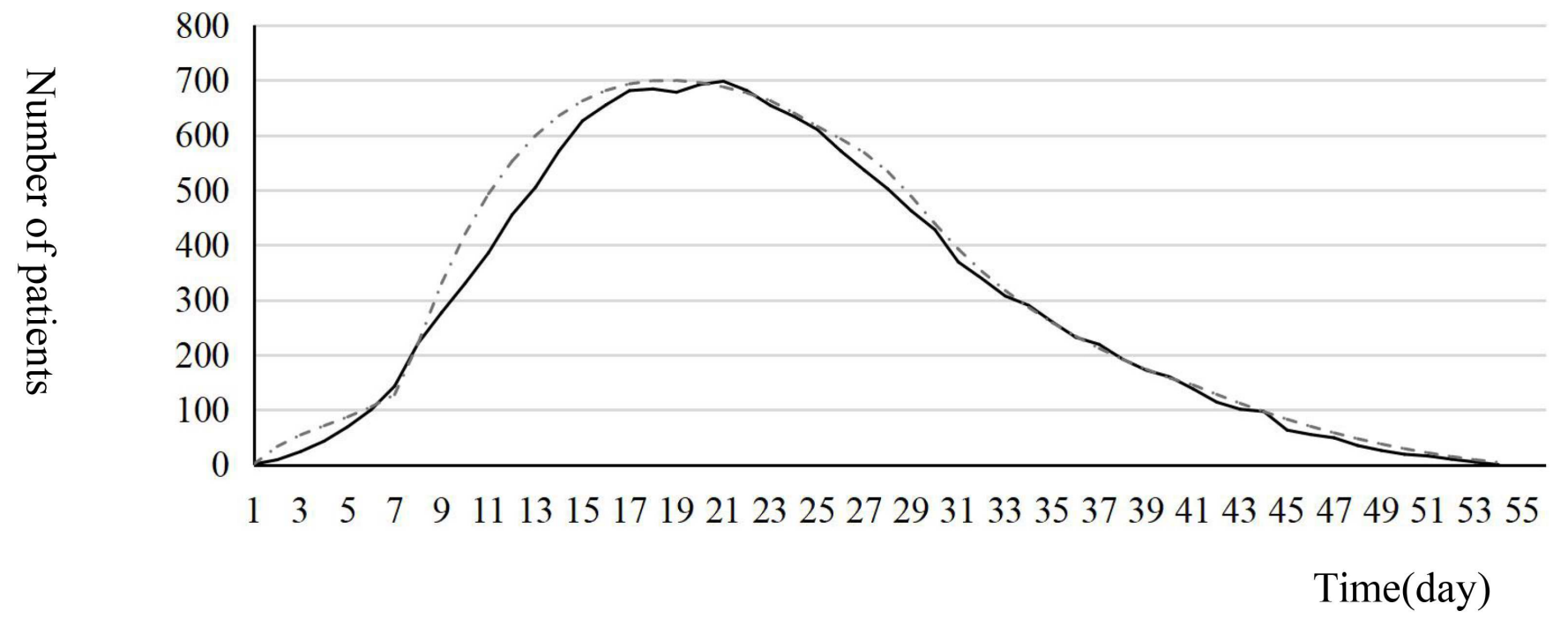

— Actual data - - - - Theoretical data

Figure 4

The result of curve fitting of actual data and SEIAR model 


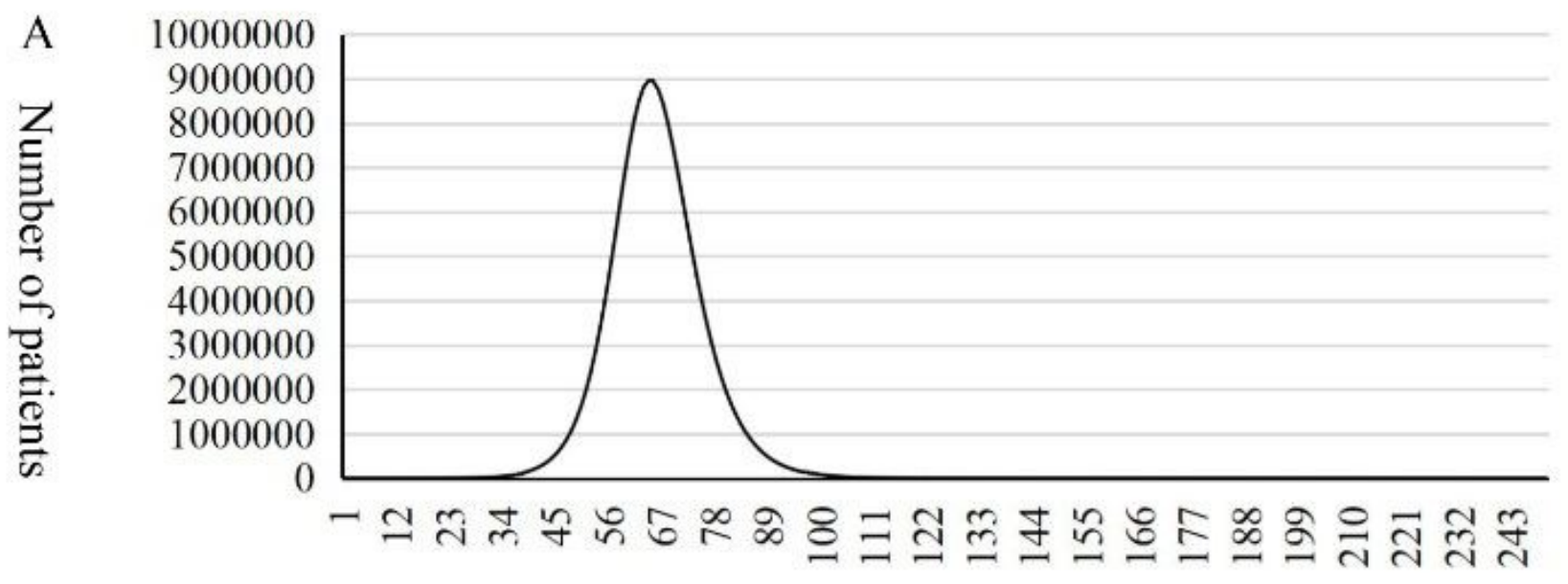

Time(day)

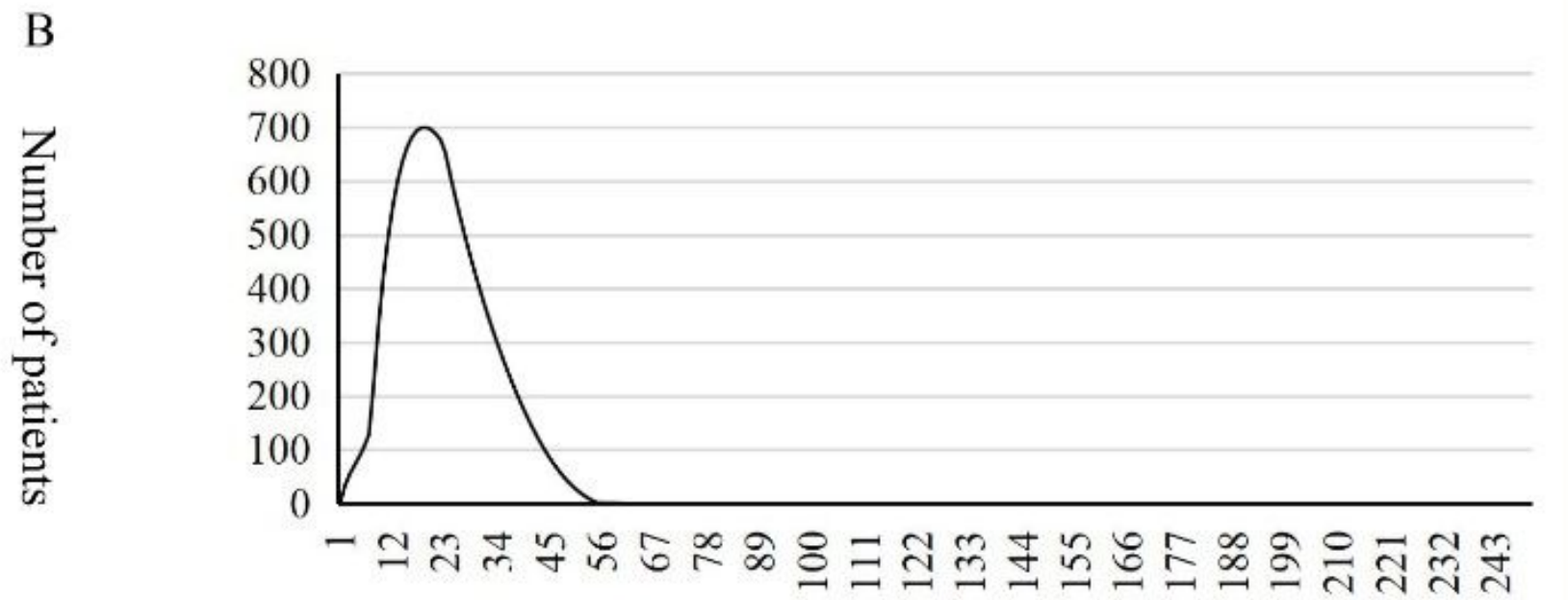

\section{Figure 5}

The forecast results with different parameters (Note: $5 \mathrm{~A}$ The forecast with parameters obtained by model fitting from January 21 to 27, 2020, when meansures have not been fully launched; 5B The forecast with parameters obtained by model fitting from January 28 to March 14, 2020, when meansures have been fully launched ) 